QUARTERLY OF APPLIED MATHEMATICS

VOLUME LXIX, NUMBER 4

DECEMBER 2011, PAGES 635-649

S 0033-569X(2011)01223-1

Article electronically published on July 7, 2011

\title{
EXISTENCE, UNIQUENESS AND EXPONENTIAL DECAY: AN EVOLUTION PROBLEM IN HEAT CONDUCTION WITH MEMORY
}

\author{
BY \\ SANDRA CARILLO \\ Dipartimento di Scienze di Base e Applicate per l'Ingegneria, Sez. Matematica SAPIENZA, Università
} di Roma, I-00161 Roma, Italy

\begin{abstract}
A rigid linear heat conductor with memory conductor is considered. An evolution problem which arises in studying the thermodynamical state of the material with memory is considered. Specifically, the time evolution of the temperature distribution within a rigid heat conductor with memory is investigated. The constitutive equations which characterize heat conduction with memory involve an integral term since the temperature's time derivative is connected to the heat flux gradient. The integrodifferential problem, when initial and boundary conditions are assigned, is studied to obtain existence and uniqueness results. Key tools turn out to be represented by suitable expressions of the minimum free energy which allow us to construct functional spaces meaningful under both the physical as well as the analytic viewpoint since therein the existence and uniqueness results can be established. Finally, conditions which guarantee exponential decay at infinity are obtained.
\end{abstract}

1. Introduction. The model of a rigid heat conductor with memory here referred to is the well-known one proposed by Gurtin and Pipkin [9], who developed results by Coleman [5] on the basis of the celebrated paper by Cattaneo [3. Subsequently, a great interest in investigating the thermodynamics of materials with memory arose. Among the many authors, results more closely related to the approach here adopted are those obtained by Coleman [5], McCarthy [10, and Coleman and Dill [6] and, in particular, by Fabrizio, Gentili and Reynolds [8], who assumed the thermodynamic state of the material to be determined when the absolute temperature $\theta$, its history $\theta^{t}$, defined via

$$
\theta^{t}(\mathbf{x}, \tau):=\theta(\mathbf{x}, t-\tau),
$$

Received February 1, 2010.

2000 Mathematics Subject Classification. Primary 80A20, 74F05.

Current address: Dipartimento di Scienze di Base e Applicate per l'Ingegneria, Sez. Matematica SAPIEnZA, 16, Via A. Scarpa, 00161 Rome, Italy.

E-mail address: carillo@dmmm.uniroma1.it

E-mail address: sandra.carillo@uniroma1.it 
and the integrated history of the temperature gradient

$$
\overline{\mathbf{g}}^{t}(\mathbf{x}, \tau):=\int_{t-\tau}^{t} \mathbf{g}(\mathbf{x}, s) d s, \mathbf{g}(\mathbf{x}, \tau):=\nabla \theta(\mathbf{x}, \tau)
$$

are known. Here, the internal energy and the relative temperature are assumed to be linearly related; that is, the internal energy $e$ reads:

$$
e(\mathbf{x}, t)=\alpha_{0} u(\mathbf{x}, t) \text { or } e(\mathbf{x}, t)=\alpha_{0}\left(\theta(\mathbf{x}, t)-\theta_{0}\right),
$$

where, for the sake of simplicity, $\alpha(\mathbf{x})=\alpha_{0}, \mathbf{x} \in \Omega \subset \mathbb{R}^{3}$ denotes the position, $t$ denotes the time variable and $u=\theta-\theta_{0}$ denotes the temperature difference between the temperature $\theta$ and $\theta_{0}$, a fixed reference temperature 1 and $\alpha_{0}>0$ is the specific heat. The constitutive equation

$$
\mathbf{q}(\mathbf{x}, t)=-\int_{0}^{\infty} k(\mathbf{x}, \tau) \nabla u(\mathbf{x}, t-\tau) d \tau
$$

relates the heat flux $\mathbf{q} \in \mathbb{R}^{3}$ with the temperature gradient, denoted as $\nabla u=\nabla\left(\theta-\theta_{0}\right)$ or $\mathbf{g}$, while $k(\mathbf{x}, \tau)$ represents the heat flux relaxation function 8 .

The latter, when the heat conductor is isotropic, depends only on the time variable, that is, $k(\mathbf{x}, \tau)=k(\tau)$; hence $k^{\prime}$ denotes its derivative. In addition,

$$
k(t)=k_{0}+\int_{0}^{t} k^{\prime}(s) d s
$$

and $k_{0} \equiv k(0)$ represents the initial value of the heat flux relaxation function, termed initial heat flux relaxation coefficient ${ }^{2}$ Indeed, crucial in this study is the dependence with respect to the time variable which is through both the present time as well as the past history. It is further required that

$$
k^{\prime} \in L^{1}\left(\mathbb{R}^{+}\right) \cap L^{2}\left(\mathbb{R}^{+}\right) \text {and } k \in L^{1}\left(\mathbb{R}^{+}\right),
$$

which imply $k(\infty):=\lim _{t \rightarrow \infty} k(t)=0$. These assumptions can be physically interpreted recalling that there is no heat flux when, at infinity, the thermal equilibrium is reached.

When the heat flux relaxation function $k$ satisfies both conditions (1.5) and (1.6), the heat flux $\mathbf{q}$ can be written in the following two equivalent forms:

$$
\mathbf{q}(t)=-\int_{0}^{\infty} k(\tau) \mathbf{g}(\mathbf{x}, t-\tau) d \tau \text { or } \mathbf{q}(t)=\int_{0}^{\infty} k^{\prime}(\tau) \overline{\mathbf{g}}^{t}(\mathbf{x}, \tau) d \tau .
$$

The evolution problem

$$
\frac{\partial e}{\partial t}=-\alpha_{0} \nabla \cdot \mathbf{q}+r \text { on } \Omega \times[0, \infty),
$$

where, in turn, $e$ is the internal energy, given by (1.3), $\mathbf{q}$ the heat flux and $r$ the external heat supply, governs the energy evolution within a rigid heat conductor.

\footnotetext{
${ }^{1}$ Here $t \in \mathbb{R}^{+}:=[0,+\infty)$ while $\theta_{0}$, according to [6], represents the temperature of a surrounding environment which is supposed not to be affected by the presence of the conductor.

${ }^{2}$ Accordingly throughout the whole paper the dependence with respect to the spatial variable $\mathbf{x}$ of the heat flux relaxation function is omitted.
} 
According to [8], the second law of thermodynamics, represented by the ClausiusDuhem inequality, reads

$$
\frac{\partial \eta}{\partial t} \geq \frac{e}{\theta}+\frac{\mathbf{q} \cdot \mathbf{g}}{\theta^{2}}
$$

where $\eta$ is the entropy and all the quantities, which depend on the space and time variables, satisfy the adopted constitutive assumptions. In the framework of the linear theory, studied in [8], the inequality (1.9) can be written as

$$
\frac{\partial \zeta}{\partial t} \leq \frac{\partial e}{\partial t} \theta+\mathbf{q} \cdot \mathbf{g}
$$

where $\zeta$ denotes the pseudo free energy 3

The thermodynamic state of the conductor with memory is chosen to be represented via the thermodynamic state function $\sigma: \mathbb{R} \rightarrow \mathbb{R} \times \mathbb{R}^{3}$ which associates $t \longmapsto \sigma(t) \equiv$ $\left(\theta(t), \overline{\mathbf{g}}^{t}\right)$; that is, the thermodynamic state is known when the temperature and the integrated history of the temperature gradient, $\overline{\mathbf{g}}^{t}$, which belongs to a suitable Hilbert space, are assigned. It is assumed that the internal energy $e$ and the free energy $\zeta$ are determined by constitutive equations of the form

$$
\begin{aligned}
& e=\hat{e}\left(\theta(\mathbf{x}, t), \overline{\mathbf{g}}^{t}(\mathbf{x}, t)\right), \\
& \zeta=\hat{\zeta}\left(\theta(\mathbf{x}, t), \overline{\mathbf{g}}^{t}(\mathbf{x}, t)\right) .
\end{aligned}
$$

In the linear case, $e$ is given by (1.3), while $\zeta$ does not assume a unique representation, but, conversely, there are many expressions of the free energy. In other words, a whole class of functionals $\hat{\zeta}$ which satisfies the inequality (1.10) can be obtained. Among them, two examples of free energies are here considered: first the functional $\zeta_{M}$ defined, see by [8], via

$$
\zeta_{M}\left(\theta, \overline{\mathbf{g}}^{t}\right):=\frac{\alpha_{0}}{2}[\theta(\mathbf{x}, t)]^{2}+\frac{1}{2} \int_{0}^{\infty} \int_{0}^{\infty} k^{\prime \prime}(\tau-\tilde{\tau}) \overline{\mathbf{g}}^{t}(\mathbf{x}, \tau) \cdot \overline{\mathbf{g}}^{t}(\mathbf{x}, \tilde{\tau}) d \tau d \tilde{\tau}
$$

then, another interesting free energy, suitable to applications, given by

$$
\zeta_{G}\left(\theta, \overline{\mathbf{g}}^{t}\right):=\frac{\alpha_{0}}{2}[\theta(\mathbf{x}, t)]^{2}-\frac{1}{2} \int_{0}^{\infty} k^{\prime}(\tau)\left(\overline{\mathbf{g}}^{t}(\mathbf{x}, \tau)\right)^{2} d \tau .
$$

The latter, when (1.3) is recalled, via derivation with respect to $t$, implies that the functional $\zeta_{G}$, termed Graff free energy, satisfies the equality

$$
\frac{\partial \zeta_{G}}{\partial t}(\mathbf{x}, t)-\frac{\partial e}{\partial t}(\mathbf{x}, t) \theta(\mathbf{x}, t)+\mathbf{q}(\mathbf{x}, t) \cdot \mathbf{g}(\mathbf{x}, t)=-\frac{1}{2} \int_{0}^{\infty} k^{\prime \prime}(\tau)\left(\overline{\mathbf{g}}^{t}(\mathbf{x}, \tau)\right)^{2} d \tau
$$

which shows that $\zeta_{G}$ represents a free energy subject to conditions (1.10) whenever $k$ satisfies the further requirements

$$
-k^{\prime}(\tau)>0, k^{\prime \prime}(\tau) \geq 0, \forall \tau \in \mathbb{R}^{+} .
$$

The latter emphasizes that the l.h.s of (1.15) is nonpositive.

The material is organized as follows. The opening Section 2 is devoted to introducing the evolution problem under investigation. In the following Section 3, existence and uniqueness results are established. Notably, such results are proved in the function space

\footnotetext{
${ }^{3}$ It is a pseudo energy since it is not dimensionally homogenous to an energy; however, as pointed out in 8, it enjoys properties which closely resemble those of the canonical free energy.
} 
which comprises all physically meaningful thermal processes, namely those associated to a finite thermal work. In the subsequent Section 4, conditions which guarantee exponential decay of solutions to the considered evolution problem are obtained on application of semigroups theory. In Section 5, on introduction of a new notion of state, the exponential decay is established in a wider function space which contains all the other spaces previously considered. This section is devoted to investigating an evolution problem which describes the temperature evolution within a rigid heat conductor with memory. That is, equation (1.8), wherein the energy $e$ is given by (1.3), subject to prescribed initial and boundary conditions, is studied. In particular, the attention is focussed on the case when the initial temperature distribution within the conductor and the temperature on the boundary are assigned.

The space-time domain, wherein the unknown function $u$ represents the temperature, is $Q_{T}=\Omega \times(0, T) \subset \mathbb{R}^{3} \times \mathbb{R}$, where $\Omega \subset \mathbb{R}^{3}$ denotes the heat conductor configuration domain. The evolution problem reads:

$$
\begin{aligned}
u_{t} & =-\nabla \cdot \mathbf{q}(\mathbf{x}, t)+r(\mathbf{x}, t), \\
\mathbf{q}(\mathbf{x}, \mathrm{t}) & =+\int_{0}^{\infty} k^{\prime}(s) \overline{\nabla u}^{t}(\mathbf{x}, s) d s
\end{aligned}
$$

when $r(\mathbf{x}, t)$ denotes the heat supply, while

$$
\overline{\nabla u}^{t}(\mathbf{x}, s):=\int_{t-s}^{t} \nabla u(\mathbf{x}, \tau) d \tau, \overline{\nabla u}^{0}(\mathbf{x}, t):=\int_{-t}^{0} \nabla u(\mathbf{x}, \tau) d \tau .
$$

The initial and boundary conditions are Dirichlet boundary conditions. On introduction of

$$
\mathbf{I}^{t}(\mathbf{x}, \tau):=\int_{0}^{\infty} k^{\prime}(\tau+s) \overline{\mathbf{g}}^{t}(\mathbf{x}, s) d s
$$

the evolution equation (1.17) reads

$$
\begin{aligned}
u_{t} & =-\nabla \int_{0}^{\infty} k(s) \overline{\nabla u}^{t}(\mathbf{x}, s) d s+r \\
& =-\nabla \int_{0}^{t} k(s) \overline{\nabla u}^{t}(\mathbf{x}, s) d s-\nabla \overline{\mathbf{I}}^{0}(\mathbf{x}, t),
\end{aligned}
$$

where the initial status and the source term, that is, the heat supply, assumed to be in gradient form, are given, in turn, by

$$
\begin{aligned}
\overline{\mathbf{I}}^{0}(\mathbf{x}, t) & :=\int_{0}^{\infty} k^{\prime}(t+\tau) \overline{\nabla u}^{0}(\mathbf{x}, \tau) d \tau, \\
r(\mathbf{x}, t) & :=-\nabla \cdot R(\mathbf{x}, t),
\end{aligned}
$$

so that the initial and boundary conditions are known when $\overline{\mathbf{I}}^{0}(\mathbf{x}, t), t \in(0, T)$, is assigned together with

$$
\begin{aligned}
u(\mathbf{x}, 0) & =u_{0}(\mathbf{x}), \\
\left.u(\mathbf{x}, t)\right|_{\partial \Omega} & =0, \quad \forall t \geq 0 .
\end{aligned}
$$


The corresponding weak formulation reads

$$
\begin{gathered}
\int_{0}^{\infty} \int_{\Omega}\left\{\alpha_{0} u_{t}(\mathbf{x}, t) \varphi(\mathbf{x}, t)+\left[k(t) \overline{\nabla u}^{t}(\mathbf{x}, t)-\int_{0}^{t} k^{\prime}(\tau) \overline{\nabla u}^{t}(\mathbf{x}, \tau) d \tau\right] \nabla \varphi(\mathbf{x}, t)\right\} d \mathbf{x} d t \\
=\int_{0}^{\infty} \int_{\Omega} \nabla \overline{\mathbf{I}}^{0}(\mathbf{x}, t) \nabla \varphi(\mathbf{x}, t) d \mathbf{x} d t+\int_{0}^{\infty} \int_{\Omega} R(\mathbf{x}, t) \nabla \varphi(\mathbf{x}, t) d \mathbf{x} d t
\end{gathered}
$$

where a subscript $t$ denotes the partial derivative with respect to the time variable while $\varphi: \Omega \times \mathbb{R} \mapsto \mathbb{R}$ is any test function.

Note that (1.22), recalling (1.19), shows that it is required to assign the thermal history only in the finite time interval $(-t, 0), t \in[0, T]$. Integration by parts allows us to write the integro-differential problem (1.25) in the equivalent form:

$$
\begin{gathered}
\int_{0}^{\infty} \int_{\Omega}\left[\alpha_{0} u_{t}(\mathbf{x}, t) \varphi(\mathbf{x}, t)-\int_{0}^{t} k(|t-\tau|) \nabla u(\mathbf{x}, \tau) \cdot \nabla \varphi(\mathbf{x}, t) d \tau\right] d \mathbf{x} d t \\
=\int_{0}^{\infty} \int_{\Omega} \mathbf{I}^{0}(\mathbf{x}, t) \nabla \varphi(\mathbf{x}, t) d \mathbf{x} d t+\int_{0}^{\infty} \int_{\Omega} R(\mathbf{x}, t) \nabla \varphi(\mathbf{x}, t) d \mathbf{x} d t
\end{gathered}
$$

where the test function $\varphi$ can be arbitrarily chosen in a suitable function space: that is, the functional space which comprises all arbitrarily chosen test functions $\varphi$ belonging to the set of all finite work processes. Indeed, the set of admissible states is represented by all those states which correspond to physically admissible processes, namely, those states which are associated to a finite thermal work. The set of all finite thermal work states, according to [2], is represented by the functional space

$$
\begin{aligned}
\mathcal{H}_{G}\left(\mathbb{R}^{+} ; H_{0}^{1}(\Omega)\right):=\left\{u \in L_{\text {loc }}^{2}\left(\mathbb{R}^{+} ; H_{0}^{1}(\Omega)\right):\right. \\
\left.\qquad\left|\int_{0}^{\infty} \int_{0}^{\infty} \int_{\Omega} k(|\tau-\tilde{\tau}|) \nabla u(\mathbf{x}, \tilde{\tau}) \cdot \nabla u(\mathbf{x}, \tau) d \mathbf{x} d \tilde{\tau} d \tau\right|<\infty\right\} .
\end{aligned}
$$

On recalling Plancherel's theorem,

$$
\begin{aligned}
\int_{0}^{\infty} \int_{0}^{\infty} \int_{\Omega} k(|\tau-\tilde{\tau}|) \nabla u(\mathbf{x}, \tilde{\tau}) & \cdot \nabla u(\mathbf{x}, \tau) d \mathbf{x} d \tilde{\tau} d \tau \\
& =\frac{1}{\pi} \int_{-\infty}^{\infty} \int_{\Omega} k_{c}(\omega) \nabla u_{+}(\mathbf{x}, \omega) \cdot \nabla u_{+}(\mathbf{x}, \omega) d \mathbf{x} d \omega
\end{aligned}
$$

where $k_{c}(\omega)$ denotes the Fourier cosine transform, see for instance [1], of $k(|\tau-s|)$, which, by definition, is an even function of its real nonnegative argument 4 and where

$$
\nabla u_{+}(\omega):=\int_{0}^{\infty} \nabla u(\mathbf{x}, \tau) e^{-i \omega \tau} d \tau
$$

\footnotetext{
${ }^{4}$ Recall that the Fourier cosine transform of the even real-valued function, see for instance [1, is real-valued; in addition, since $k_{c}(0)>0$, as prescribed by the adopted assumptions 8 and the thermodynamical restrictions on the constitutive equation, $k_{c}(\omega)$ is positive-valued.
} 
denotes the half-line Fourier transforms of $\nabla u(\mathbf{x}, \tau)$. Hence, the function space $\mathcal{H}_{G}\left(\mathbb{R}^{+}\right.$; $\left.H_{0}^{1}(\Omega)\right)$ can be characterized as

$$
\begin{aligned}
\mathcal{H}_{G}\left(\mathbb{R}^{+} ; H_{0}^{1}(\Omega)\right):=\{u & \in L_{l o c}^{2}\left(\mathbb{R} ; H_{0}^{1}(\Omega)\right): \\
& \left.=\frac{1}{\pi}\left|\int_{-\infty}^{\infty} \int_{\Omega} k_{c}(\omega) \nabla u_{+}(\mathbf{x}, \omega) \cdot \nabla u_{+}(\mathbf{x}, \omega) d \mathbf{x} d \omega\right|<\infty\right\} .
\end{aligned}
$$

Precisely in such a functional space the evolution equation (1.21), together with the initial boundary conditions (1.22) - (1.24), admits a unique solution according to the results comprised in the following section. Furthermore, the function space $\mathcal{H}_{G}$ is a Hilbert space when equipped with the inner product

$$
\langle f, \varphi\rangle_{\mathcal{H}_{G}}:=\int_{-\infty}^{+\infty} \int_{\Omega} k_{c}(\omega) \nabla f_{+}(\mathbf{x}, \omega) \cdot \overline{\nabla \varphi_{+}(\mathbf{x}, \omega)} d \mathbf{x} d \omega
$$

and the induced norm

$$
\|\varphi\|_{\mathcal{H}_{G}}:=\langle\varphi, \varphi\rangle_{\mathcal{H}_{G}}=\int_{-\infty}^{+\infty} \int_{\Omega} k_{c}(\omega) \nabla \varphi_{+}(\mathbf{x}, \omega) \cdot \overline{\nabla \varphi_{+}(\mathbf{x}, \omega)} d \mathbf{x} d \omega .
$$

Such definitions follow from formulae (3.20) and (3.21) in [2], when the dependence on the spatial $\mathrm{x}$-variable is recovered and, in addition, the function space which comprises all admissible thermal states is explicitly represented in terms of the temperature distribution $u: \Omega \times(0, T) \subset \mathbb{R}^{3} \times \mathbb{R} \rightarrow \mathbb{R}$ instead of its gradient and the related integrated history which appear in [2. Notably, there are interesting analogies, as far as the function spaces which contain physically meaningful states of the system are concerned, between the case of a rigid heat conductor with memory and an isothermal viscoelastic body, according to results in [4].

2. Existence and uniqueness result. The aim of this section is to prove that the evolution problem under investigation admits a unique solution when the simplifying assumption that there is no heat supply is adopted.

First of all, it is shown that the evolution problem is related, via duality under Fourier transform, to an equivalent elliptic problem. The latter is proved to enjoy the coercivity property; hence, existence and uniqueness of the solution follow from known results.

The arbitrariness of the test function $\varphi$ in the weak formulation (1.26) of the evolution problem (1.21) - (1.24) imply the equivalence between the weak problem and the following one:

$$
u_{t}(\mathbf{x}, t)=\nabla \cdot \int_{0}^{t} k(t-\tau) \nabla u(\mathbf{x}, \tau) d \tau-\nabla \cdot \mathbf{I}^{0}(\mathbf{x}, t),
$$

where $\mathbf{I}^{0}$ is assigned together with the initial and boundary conditions

$$
u(\mathbf{x}, 0)=u_{0}(\mathbf{x}),\left.u(\mathbf{x}, t)\right|_{\partial \Omega}=0,
$$

and the unknown temperature is now denoted by $u$.

Problem (2.1), subject to the initial and boundary conditions (2.2), on application of the Fourier transform, is related, via duality, to the following:

$$
-i \omega u_{+}(\mathbf{x}, \omega)+\nabla \cdot\left[k_{+}(\omega) \nabla u_{+}(\mathbf{x}, \omega)\right]=\nabla \mathbf{I}_{+}^{0}(\mathbf{x}, \omega)
$$


while the initial and boundary conditions (2.2) now read:

$$
u(\mathbf{x}, 0)=u_{0}(\mathbf{x}),\left.u_{+}(\mathbf{x}, \omega)\right|_{\partial \Omega}=0,
$$

where

$$
\mathbf{I}_{+}^{0}(\mathbf{x}, \omega):=\int_{0}^{\infty} \mathbf{I}^{0}(\mathbf{x}, \tau) e^{-i \omega \tau} d \tau, u_{+}(\mathbf{x}, \omega):=\int_{0}^{\infty} u(\mathbf{x}, \tau) e^{-i \omega \tau} d \tau
$$

denote, in turn, the half-line Fourier transforms of the assigned data $\mathbf{I}^{0}(\mathbf{x}, \tau)$ and of the unknown $u(\mathbf{x}, \tau)$.

Hence, as soon as a solution of the problem (2.3)-(2.4) is known, a solution of the original problem (1.21), which satisfies the initial and boundary conditions (1.23)-(1.24), is obtained. Accordingly, existence and uniqueness results can be stated as far as the problem (2.3)-(2.4) is concerned.

Theorem 2.1. If $k_{c}(\omega)>0 \forall \omega \in \mathbb{R}$, then the initial boundary value problem (2.3), subject to the initial and boundary conditions (2.4), admits a unique solution.

Proof. The proposition is proved when it is shown that (2.3) enjoys the coercivity property. Thus, consider the bilinear form

$$
a\left(u_{+}(\omega), u_{+}(\omega)\right):=\int_{\Omega}\left[i \omega u_{+}(\mathbf{x}, \omega) \cdot \overline{u_{+}(\mathbf{x}, \omega)}+k_{c}(\omega) \nabla u_{+}(\mathbf{x}, \omega) \cdot \overline{\nabla u_{+}(\mathbf{x}, \omega)}\right] d \mathbf{x} .
$$

It is bounded for any fixed $\omega \in \mathbb{R}$ and for any fixed $u_{+}(\cdot, \omega) \in H_{0}^{1}(\Omega)$; indeed, when $H_{0}^{1}(\Omega)$ is equipped with the norm

$$
\|\varphi\|_{H_{0}^{1}}:=\left(\int_{-\infty}^{+\infty} \int_{\Omega}\left|\varphi_{+}(\mathbf{x}, \omega)\right|^{2}+\left|\nabla \varphi_{+}(\mathbf{x}, \omega)\right|^{2} d \mathbf{x} d \omega\right)^{\frac{1}{2}},
$$

then coercivity follows since, $\forall \omega \in \mathbb{R}$, the adopted thermodynamical assumptions imply that $k(\omega)$ is a positive real constant and, in addition, definition (2.6) implies that, $\forall \mathbf{x} \in$ $\Omega, \forall u_{+}(\mathbf{x}, \omega) \in H_{0}^{1}(\Omega)$, the following inequality

$$
\left|a\left(u_{+}(\omega), u_{+}(\omega)\right)\right| \geq k(\omega)\left\|u_{+}(\mathbf{x}, \omega)\right\|_{H_{0}^{1}}^{2}
$$

is satisfied.

For any fixed $\omega \neq 0$, the problem (2.3) $-(2.4)$ admits a solution $u_{+} \in H_{0}^{1}(\Omega)$, whenever $\nabla \cdot \mathbf{I}_{+}^{0}(\mathbf{x}, \omega) \in H^{-1}(\Omega)$. Now let

$$
\mathcal{L}\left(\mathbf{I}^{0}, \nabla u\right):=\int_{0}^{\infty} \int_{\Omega} \mathbf{I}^{0}(\mathbf{x}, \tau) \cdot \nabla u(\mathbf{x}, \tau) d \mathbf{x} d \tau
$$

define a linear functional on $\mathcal{H}_{G}\left(\mathbb{R}^{+} ; H_{0}^{1}(\Omega)\right)$. The Riesz theorem [11] implies the existence of an element $\nabla u^{\mathbf{I}^{0}} \in \mathcal{H}^{\prime}{ }_{G}\left(\mathbb{R}^{+} ; H_{0}^{1}(\Omega)\right)$ such that $\forall \nabla u(\mathbf{x}, \tau) \in \mathcal{H}_{G}\left(\mathbb{R}^{+} ; H_{0}^{1}(\Omega)\right)$,

$$
\mathcal{L}\left(\mathbf{I}^{0}, \nabla u\right)=\int_{0}^{\infty} \int_{0}^{\infty} \int_{\Omega} k(\mathbf{x},|\tau-\tilde{\tau}|) \nabla u^{\mathbf{I}^{0}}(\mathbf{x}, \tilde{\tau}) \cdot \nabla u(\mathbf{x}, \tau) d \mathbf{x} d \tilde{\tau} d \tau .
$$

Hence, let

$$
\mathbf{F}^{0}(\mathbf{x}, \tau):=\int_{0}^{\infty} k(\mathbf{x},|\tau-\tilde{\tau}|) \nabla u^{\mathbf{I}^{0}}(\mathbf{x}, \tilde{\tau}) d \tilde{\tau}, \tau \in \mathbb{R}
$$


which, by definition, is unique when $\mathbf{I}^{0}(\mathbf{x}, \tau)$ has been arbitrarily chosen; that is, there is only one function $\mathbf{F}^{0}(\mathbf{x}, \tau)$, given by (2.11), such that, for fixed $\tau \in \mathbb{R}^{+}$, it turns out that $\mathbf{F}^{0}(\mathbf{x}, \tau)=\mathbf{I}^{0}(\mathbf{x}, \tau), \forall \mathbf{x} \in \Omega$. Integration over both time and space variables delivers

$$
\mathcal{L}\left(\mathbf{I}^{0}, \nabla u\right)=\int_{0}^{\infty} \int_{\Omega} \mathbf{I}^{0}(\mathbf{x}, \tau) \cdot \nabla u(\mathbf{x}, \tau) d \mathbf{x} d \tau=\int_{0}^{\infty} \int_{\Omega} \mathbf{F}^{0}(\mathbf{x}, \tau) \cdot \nabla u(\mathbf{x}, \tau) d \mathbf{x} d \tau
$$

for all $u(\mathbf{x}, \tau) \in \mathcal{H}_{G}\left(\mathbb{R}^{+} ; H_{0}^{1}(\Omega)\right)$. Notably, the same procedure allows us to prove existence and uniqueness of the solution in the case of an initial boundary value problem in which conditions of no heat flux on the boundary are imposed.

3. Semigroup theory. In this section the evolution problem is analyzed under the semigroup approach viewpoint. Specifically, the dependence with respect to the two space and time variables is considered in a fairly different way. Namely, to apply results of Datko and Pazy [7, 12, the evolution problem is modeled as an abstract dynamical system; that is, it is rewritten under the abstract form

$$
\chi_{t}=A \chi, \chi(0)=\chi_{0},
$$

where $A$ denotes the operator represented by the right-hand side of (1.17)-(1.18), written in the form of a system

$$
\left\{\begin{array}{l}
u_{t}(\mathbf{x}, t)=\nabla \cdot \int_{0}^{+\infty} k^{\prime}(\tau) \overline{\mathbf{g}}^{t}(\mathbf{x}, \tau) d \tau, \\
\overline{\mathbf{g}}_{t}^{t}(\mathbf{x}, \tau)=\nabla u(\mathbf{x}, t)-\nabla u(\mathbf{x}, t-\tau),
\end{array}\right.
$$

where $r=0$; i.e., no heat source is assumed. Comparison between (3.1) and (3.2) shows that, in the latter, the state $\chi$ is given by the pair $\chi(\mathbf{x}, t) \equiv\left(u(\mathbf{x}, t), \overline{\mathbf{g}}^{t}(\mathbf{x}, \cdot)\right)$. The system (3.2) is supplemented by the Dirichlet boundary condition

$$
u(\mathbf{x}, t)=0 \forall \mathbf{x} \in \partial \Omega .
$$

When the Graffi free energy $\zeta_{G}$ in (1.14) is considered, restrictions (1.16), namely

$$
0<-k^{\prime}(\tau) \leq \delta k^{\prime \prime}(\tau), k^{\prime}(0) \text { bounded }, \delta \in \mathbb{R}^{+} \text {constant },
$$

need to be imposed on the kernel $k^{\prime}(\tau)$. Thus, the state $\chi$ turns out to belong to the Hilbert space $\mathcal{H}$ defined by the inner product ${ }^{5}$

$$
\left\langle\chi_{1}(t), \chi_{2}(t)\right\rangle:=\frac{1}{2} \int_{\Omega} \alpha_{0} u_{1}(\mathbf{x}, t) u_{2}(\mathbf{x}, t) d \mathbf{x}-\frac{1}{2} \int_{\Omega} \int_{0}^{\infty} k^{\prime}(\tau) \overline{\mathbf{g}}_{1}^{t}(\mathbf{x}, \tau) \cdot \overline{\mathbf{g}}_{2}^{t}(\mathbf{x}, \tau) d \tau d \mathbf{x}
$$

that induces the norm

$$
\|\chi(t)\|^{2}:=\langle\chi(t), \chi(t)\rangle
$$

${ }^{5}$ Such an inner product can be generalized to give

$$
\left\langle\chi_{1}(t), \chi_{2}(t)\right\rangle:=\frac{1}{2} \int_{\Omega} \alpha(\mathbf{x}) u_{1}(\mathbf{x}, t) u_{2}(\mathbf{x}, t) d \mathbf{x}-\frac{1}{2} \int_{\Omega} \int_{0}^{\infty} k^{\prime}(\tau) \overline{\mathbf{g}}_{1}^{t}(\mathbf{x}, \tau) \cdot \overline{\mathbf{g}}_{2}^{t}(\mathbf{x}, \tau) d \tau d \mathbf{x}
$$

when the assumption $\alpha(\mathbf{x})=\alpha_{0}$ is removed. 
which represents the energy associated to the system. Accordingly, the energy associated to the rigid heat conductor can be written in the form

$$
\mathcal{E}(t)=\frac{1}{2} \int_{\Omega} \alpha_{0}[u(\mathbf{x}, t)]^{2} d \mathbf{x}-\frac{1}{2} \int_{\Omega} \int_{0}^{\infty} k^{\prime}(\tau)\left[\overline{\mathbf{g}}^{t}(\mathbf{x}, \tau)\right]^{2} d \tau d \mathbf{x} .
$$

When (3.4) is satisfied, solutions to (3.2)-(3.3) exhibit an exponential decay at infinity.

Lemma 3.1. Under the hypothesis (3.4), the total energy associated to the system (3.2) - (3.3) is integrable on $[0, \infty)$.

Proof. Let $\chi(t)$ denote a solution of the evolution problem (3.2) - (3.3), equivalent to (1.17) - (1.18); on substitution of such a solution in (1.15), integration over the whole space domain $\Omega$ shows that the time derivative of the free energy associated to the rigid heat conductor is nonincreasing; indeed,

$$
\int_{\Omega} \frac{\partial \zeta_{G}}{\partial t} d \mathbf{x}=-\frac{1}{2} \int_{\Omega} \int_{0}^{\infty} k^{\prime \prime}(\tau)\left[\overline{\mathbf{g}}^{t}(\mathbf{x}, \tau)\right]^{2} d \tau d \mathbf{x}
$$

where the r.h.s. is negative, as follows from (3.4). On the other hand, by definition (3.8), the initial value of the energy is given by

$$
\mathcal{E}(0)=\frac{1}{2} \int_{\Omega} \alpha_{0}\left[u_{0}(\mathbf{x})\right]^{2} d \mathbf{x}-\frac{1}{2} \int_{\Omega} \int_{0}^{\infty} k^{\prime}(\tau)\left[\overline{\mathbf{g}}^{0}(\mathbf{x}, \tau)\right]^{2} d \tau d \mathbf{x} .
$$

Hence, the energy $\mathcal{E}(t)$, given by (3.8), since $\frac{\partial \mathcal{E}}{\partial t}<0$, will then satisfy

$$
0 \leq \mathcal{E}(t) \leq \mathcal{E}(0)
$$

Moreover (3.9), on integration over $[0, \infty)$, recalling 3.4, gives

$$
\int_{0}^{\infty} \int_{\Omega} \frac{\partial \zeta_{G}}{\partial t} d \mathbf{x} d t=-\frac{1}{2} \int_{0}^{\infty} \int_{\Omega} \int_{0}^{\infty} k^{\prime \prime}(\tau)\left[\overline{\mathbf{g}}^{t}(\mathbf{x}, \tau)\right]^{2} d \tau d \mathbf{x} d t<0
$$

which implies

$$
-\int_{0}^{\infty} \int_{\Omega} \int_{0}^{\infty} k^{\prime \prime}(\tau)\left[\overline{\mathbf{g}}^{t}(\mathbf{x}, \tau)\right]^{2} d \tau d \mathbf{x} d t<\infty .
$$

Finally, the definition of the total energy $\mathcal{E}(t)$, together with Theorem 4.3 in [8], allows us to write

$$
2 \int_{0}^{\infty} \mathcal{E}(t) d t=\int_{0}^{\infty}\left\{\int_{\Omega} \alpha_{0}[u(\mathbf{x}, t)]^{2} d \mathbf{x}-\int_{\Omega} \int_{0}^{\infty} k^{\prime}(u)\left[\overline{\mathbf{g}}^{t}(\mathbf{x}, \tau)\right]^{2} d \tau d \mathbf{x}\right\} d t<\infty .
$$

The operator $A$, represented by the right-hand side of (3.2)-(3.3), is defined over the domain

$$
\mathcal{D}_{G}=\left\{\chi \in \mathcal{H} ; u(t) \in H_{0}^{1}(\Omega), \overline{\mathbf{g}}^{t} \in L^{2}(\Omega):\left|\int_{0}^{\infty} \int_{\Omega} k^{\prime}(\tau)\left[\overline{\mathbf{g}}^{t}(\mathbf{x}, \tau)\right]^{2} d \tau d \mathbf{x}\right|<\infty\right\},
$$

where the subscript ${ }_{G}$ reminds us of its connection with Graffi's free energy.

Lemma 3.2. Under the same hypothesis of Lemma 3.1, the operator $A: \mathcal{D}_{G} \rightarrow \mathcal{H}$ is a maximal dissipative operator on $\mathcal{H}$, i.e.

i. $\langle A \chi, \chi\rangle \leq 0, \forall \chi \in \mathcal{D}_{G}$;

ii. the range of $A-I$, when $I$ denotes the identity operator, coincides with $\mathcal{H}$. 
Proof. Lemma 3.2, together with Lurm-Phillips' theorem, see Pazy [12, implies that the operator $A$ generates a strongly continuous contraction semigroup $S(t)$ on $\mathcal{H}$, so that the solution to (3.1) can be written in the form $\chi(t)=S(t) \chi_{0}$. Then, the energy reads

$$
\mathcal{E}(t)=\frac{1}{2}\left\langle S(t) \chi_{0}, S(t) \chi_{0}\right\rangle,
$$

and the inequality (3.14) implies that

$$
\int_{0}^{\infty}\left\langle S(t) \chi_{0}, S(t) \chi_{0}\right\rangle d t<\infty, \forall \chi_{0} \in \mathcal{H} .
$$

TheOREm 3.3. Under the same hypothesis of Lemma 3.1, there exist two positive real constants $M$ and $\gamma$ such that

$$
\mathcal{E}(t) \leq M e^{-\gamma t} \mathcal{E}(0), \forall \chi_{0} \in \mathcal{H} .
$$

Proof. The proof is a direct application of the following lemma.

LEmma 3.4. Let $M$ and $\gamma$ be positive constants. A strongly continuous semigroup of linear operators $S(t)$, defined on $\mathcal{H}$, satisfies the inequality

$$
\left\langle S(t) \chi_{0}, S(t) \chi_{0}\right\rangle \leq M e^{-\gamma t}\left\langle\chi_{0}, \chi_{0}\right\rangle, \forall \chi_{0} \in \mathcal{H},
$$

if and only if

$$
\int_{0}^{\infty}\left\langle S(t) \chi_{0}, S(t) \chi_{0}\right\rangle \text { converges } \forall \chi_{0} \in \mathcal{H} \text {. }
$$

By definition, $\left\langle\chi_{0}, \chi_{0}\right\rangle=2 \mathcal{E}(0)$ and $\left\langle S(t) \chi_{0}, S(t) \chi_{0}\right\rangle=2 \mathcal{E}(t)$. Then (3.17) implies (3.18).

4. Free energy for the new notion of state. The aim of this section is to reformulate the evolution problem under investigation in terms of the functional $\mathbf{I}^{t}(\tau)$ which can be used to describe the status of the system and, in particular, $\mathbf{I}^{0}$ represents the initial datum, at initial time $t=0$. To better understand the meaning of this choice, some remarks concerning the thermodynamical status of the rigid heat conductor and how it can be represented via the functional $\mathbf{I}^{t}(\tau)$ are given. The functional $\mathbf{I}^{t}(\tau)$ depends on the thermal state of the conductor via $\overline{\mathbf{g}}^{t}(\cdot)$. Thus, it should be, more appropriately, denoted as $\mathbf{I}\left(\tau, \overline{\mathbf{g}}^{t}\right)$; however, this notation is used only to emphasize the dependence on $\overline{\mathbf{g}}^{t}(\cdot)$, when it is crucial. Accordingly, first of all, the notion of equivalence between different thermal states is reformulated in terms of the quantity $\mathbf{I}^{t}(\tau)$. Then, also, the thermal work is expressed as a function of $\mathbf{I}^{t}(\tau)$; indeed, in such a way, recalling that whenever two different thermal states are equivalent, they are associated to the same thermal work, a further relation which characterizes equivalent thermal states is obtained. Then, a new form of the minimum free energy, which depends on $\mathbf{I}^{t}(\tau)$, follows. The section closes by showing that the function space which comprises all admissible states, characterized as those states associated to a finite $\mathbf{I}^{t}(\tau)$, contains both the set of states associated to the free energies $\zeta_{M}$ as well as $\zeta_{G}$. For the sake of simplicity, in this section, the 
$\mathbf{x}$-dependence is omitted. Indeed, the attention is focussed on the time dependence and, hence, the spatial variable $\mathbf{x}$ plays the role of a parameter in the whole section.

The notion of equivalence between two different thermal states, according to [2, can be formulated in terms of the heat flux or of the thermal work. Indeed, any two different thermal states are termed equivalent [2] whenever the thermal work associated to both of them is the same. Equivalence between any two different thermal processes can be, also, stated by referring to the quantity $\mathbf{I}^{t}(\tau)$, as follows.

Definition 4.1. Two arbitrary histories of the temperature-gradient, corresponding to the states $\left(\theta_{1}(t), \overline{\mathbf{g}}_{1}^{t}\right)$ and $\left(\theta_{2}(t), \overline{\mathbf{g}}_{2}^{t}\right)$, are equivalent if and only if

$$
\left\{\begin{array}{l}
\theta_{1}(t)=\theta_{2}(t), \\
\mathbf{I}\left(\tau, \overline{\mathbf{g}}_{1}^{t}\right)=\mathbf{I}\left(\tau, \overline{\mathbf{g}}_{2}^{t}\right) .
\end{array}\right.
$$

In addition, according to [2], the definition of prolongation, which prescribes how to construct a continuos prolongation of the integrated history via an assigned process $P$, is here recalled as follows.

Definition 4.2. Given a process $P$, hence assigned $\mathbf{g}_{P}$, its duration $T$ and a set of integrated histories of the temperature gradient $\overline{\mathbf{g}}_{i}^{t}(s)$, corresponding to $\mathbf{g}_{i}^{t}(s), i=$ $1, \ldots, n, n \in \mathbb{N}$, then the prolongation of the history $\overline{\mathbf{g}}_{i}^{t}(s)$, induced by the process $P$, is defined by

$$
\left(\mathbf{g}_{P} \star \overline{\mathbf{g}}_{i}\right)^{t+T}(s):=\left\{\begin{array}{lc}
\overline{\mathbf{g}}_{P}^{T}(s)=\int_{T-s}^{T} \mathbf{g}_{P}(\xi) d \xi & 0 \leq s<T, \\
\left.\overline{\mathbf{g}}_{P}^{T}(T)+\overline{\mathbf{g}}_{i}^{t}(s-T)=\int_{0}^{T} \mathbf{g}_{P}(\xi) d \xi+\int_{t+T-s}^{t} \mathbf{g}_{i}(\xi) d \xi s\right) d \tilde{s} & s \geq T .
\end{array}\right.
$$

Proposition 4.1. Two equivalent states are associated to the same thermal work.

Proof. In [2], it is shown that the thermal work can be written as

$$
\widetilde{W}\left\{\overline{\mathbf{g}}^{t} ; \mathbf{g}_{P}\right\}=\int_{0}^{\infty}\left[\frac{1}{2} \int_{0}^{\infty} k(|\tau-s|) \mathbf{g}_{P}(s) d s-\mathbf{I}\left(\tau, \overline{\mathbf{g}}^{t}\right)\right] \cdot \mathbf{g}_{P}(\tau) d \tau .
$$

Hence, $\forall \mathbf{g}_{P}:[0, \tau) \rightarrow \mathbb{R}^{3}, \forall \tau>0$, the arbitrariness of $\tau$ and of the process $\mathbf{g}_{P}$ completes the proof; namely,

$$
\mathbf{I}\left(\tau, \overline{\mathbf{g}}_{1}^{t}\right)=\mathbf{I}\left(\tau, \overline{\mathbf{g}}_{2}^{t}\right) \Longleftrightarrow \widetilde{W}\left\{\overline{\mathbf{g}}_{1}^{t} ; \mathbf{g}_{P}\right\}=\widetilde{W}\left\{\overline{\mathbf{g}}_{2}^{t} ; \mathbf{g}_{P}\right\}
$$

Proposition 4.1 implies that when the functional $\mathbf{I}^{t}(\tau)$ is assigned, the minimal information on the thermodynamical state of the heat conductor is known. Indeed, since all the thermal histories characterized by the same $\mathbf{I}^{t}(\tau)$ are equivalent, the state of the heat conductor is completely assigned by the pair $\left(u, \overline{\mathbf{g}}^{t}\right)$ or, in a completely equivalent manner, by the pair $\left(u, \mathbf{I}^{t}(\tau)\right)$. Consider again the evolution problem (3.2)-(3.3). It can be written under the equivalent form

$$
\mathbf{q}(t)=\int_{0}^{t} k^{\prime}(\tau) \overline{\mathbf{g}}^{t}(\tau) d \tau-\mathbf{I}^{0}(t),
$$


where, according to [8], the new notion of minimal state is defined via

$$
\mathbf{I}^{0}(\tau):=\int_{0}^{\infty} k^{\prime}(\tau+s) \overline{\mathbf{g}}^{0}(s) d s
$$

that is, since $\mathbf{I}^{t}(\tau)$ characterizes the minimal information required to identify the state of the material, it replaces the past history $\overline{\mathbf{g}}^{0}(\tau)$.

Now, it is worth noting that via the notion of the state $\mathbf{I}^{t}(\tau)$, it is possible to consider a new free energy given by the functional

$$
\Psi_{I}:=-\frac{1}{2} \int_{0}^{\infty}\left[k^{\prime}(\tau)\right]^{-1}\left\{\left[\mathbf{I}^{t}(\tau)\right]^{\prime}\right\}^{2} d \tau,\left[\mathbf{I}^{t}(\tau)\right]^{\prime}:=\frac{d}{d \tau} \mathbf{I}^{t}(\tau)
$$

which, after derivation with respect to $t$, via integration by parts combined with the definitions of $\mathbf{I}^{t}(\tau)$ and of $\mathbf{q}(t)$, in turn, (1.20) and (1.7), gives

$$
\dot{\Psi}_{I} \leq \mathbf{I}^{t}(0) \cdot \mathbf{g}(t), \mathbf{I}^{t}(0) \cdot \mathbf{g}(t)=\mathbf{q}(t) \cdot \mathbf{g}(t) .
$$

Indeed, the latter follows by recalling definition (1.20) of $\mathbf{I}^{t}(\tau)$, where substitution of $\tau=0$ shows that $\mathbf{I}^{t}(0)=q(t)$. In addition, definition $(1.20)$, when $\dot{\mathbf{I}}^{t}(\tau):=\frac{d}{d t} \mathbf{I}^{t}(\tau)$, implies that

$$
\dot{\mathbf{I}}^{t}(\tau)=-k(\tau) \mathbf{g}(t)+\int_{0}^{\infty} k^{\prime \prime}(\tau+s) \overline{\mathbf{g}}^{t}(s) d s
$$

and

$$
\ddot{\mathbf{I}}^{t}(\tau)=-k^{\prime}(\tau) \mathbf{g}(t)+\int_{0}^{\infty} k^{\prime \prime \prime}(\tau+s) \overline{\mathbf{g}}^{t}(s) d s
$$

that is,

$$
\dot{\mathbf{I}}^{t}(\tau)=-k(\tau) \mathbf{g}(t)+\left[\mathbf{I}^{t}(\tau)\right]^{\prime}
$$

and

$$
\ddot{\mathbf{I}}^{t}(\tau)=-k^{\prime}(\tau) \mathbf{g}(t)+\left[\mathbf{I}^{t}(\tau)\right]^{\prime \prime},
$$

where the definition (4.7) of derivation with respect to $\tau$ has been substituted, giving

$$
\left[\mathbf{I}^{t}(\tau)\right]^{\prime}=\int_{0}^{\infty} k^{\prime \prime}(\tau+s) \overline{\mathbf{g}}^{t}(s) d s,\left[\mathbf{I}^{t}(\tau)\right]^{\prime \prime}=\int_{0}^{\infty} k^{\prime \prime \prime}(\tau+s) \overline{\mathbf{g}}^{t}(s) d s .
$$

Accordingly, the latter is obtained in the case when $k(\tau)$ satisfies further regularity requirements; in particular, it is assumed that $k^{\prime \prime}(\tau) \in L^{1}\left(\mathbb{R}^{+}\right)$. Hence, when the derivative with respect to $t$ of the free energy $\Psi_{I}$, given by (4.7), is evaluated, since, by definition (1.20), $\mathbf{I}^{t}(0)=\mathbf{q}(t)$, it follows that

$$
\begin{aligned}
\frac{d}{d t} \Psi_{I} & =-\frac{1}{2} \int_{0}^{\infty}\left[k^{\prime}(\tau)\right]^{-1} \frac{d}{d t}\left\{\left[\mathbf{I}^{t}(\tau)\right]^{\prime} \cdot\left[\mathbf{I}^{t}(\tau)\right]^{\prime}\right\} d \tau \\
& =-\int_{0}^{\infty}\left[k^{\prime}(\tau)\right]^{-1}\left\{\left[\mathbf{I}^{t}(\tau)\right]^{\prime \prime}-k(\tau) \nabla u(\mathbf{x}, t)\right\} \cdot\left[\mathbf{I}^{t}(\tau)\right]^{\prime} d \tau
\end{aligned}
$$

so that

$$
\frac{d}{d t} \Psi_{I}=\mathbf{q}(t) \cdot \mathbf{g}(t)-\int_{0}^{\infty}\left[k^{\prime}(\tau)\right]^{-1}\left[\mathbf{I}^{t}(\tau)\right]^{\prime \prime} \cdot\left[\mathbf{I}^{t}(\tau)\right]^{\prime} d \tau .
$$

Finally, for any kernel $k$ which satisfies (3.4), it follows that

$$
\int_{0}^{\infty}\left[k^{\prime}(\tau)\right]^{-1}\left[\mathbf{I}^{t}(\tau)\right]^{\prime \prime} \cdot\left[\mathbf{I}^{t}(\tau)\right]^{\prime} d \tau \geq 0
$$


which implies the inequality

$$
\frac{d}{d t} \Psi_{I} \leq-\alpha_{0} \Psi_{I}+\mathbf{q}(t) \cdot \mathbf{g}(t)
$$

hence, it follows that

$$
\frac{d}{d t} \Psi_{I}<0 .
$$

The advantage of the use of this new free energy $\Psi_{I}$ is given by its large domain of definition:

$$
\mathcal{D}_{I}:=\left\{\chi \in \mathcal{H} ; u(t) \in H_{0}^{1}(\Omega), \mathbf{I}^{t} \in L^{2}(\Omega):\left|\int_{0}^{\infty} \int_{\Omega}\left(k^{\prime}(\tau)\right)^{-1}\left\{\left[\mathbf{I}^{t}(\tau)\right]^{\prime}\right\}^{2} d \tau\right|<\infty\right\} ;
$$

indeed,

$$
\mathcal{D}_{I} \supset \mathcal{D}_{G} \supset \mathcal{D}_{M}
$$

where $\mathcal{D}_{M}$ denotes the function space which comprises all the states associated to a finite free energy $\zeta_{M}$ given by (1.13), defined in 8]. In this framework, the state $\sigma$, which characterizes the thermodynamical status of the rigid heat conductor, is defined by the pair

$$
\sigma(t) \equiv\left(u(t), \mathbf{I}^{t}(\cdot)\right)
$$

written in terms of the temperature $u$. Hence, the dynamical system (3.2) can be represented by the differential system

$$
\left\{\begin{array}{l}
\dot{u}(\mathbf{x}, t)=\nabla \cdot \mathbf{I}^{t}(\tau), \\
\dot{\mathbf{I}}^{t}(\tau)=\left[\mathbf{I}^{t}(\tau)\right]^{\prime}-k(\tau) \nabla u(\mathbf{x}, t),
\end{array}\right.
$$

where, once again, the space variable $\mathbf{x}$ plays the role of a parameter. Exponential decay follows on use of the same arguments already given in Section 4.

The system (4.21) is subject to the initial condition $\sigma(0)=\sigma_{0}$; namely,

$$
\left\{\begin{array}{l}
\left.u(\mathbf{x}, t)\right|_{t=0}=u_{0}(\mathbf{x}), \\
\left.\mathbf{I}^{t}(\tau)\right|_{t=0}=\mathbf{I}^{0}(\tau) .
\end{array}\right.
$$

The function space $\mathcal{D}_{I}$, wherein we look for solutions to (4.21)-(4.22), can be equipped with the inner product

$$
\left\langle\sigma_{1}(t), \sigma_{2}(t)\right\rangle:=\frac{1}{2} \int_{\Omega} \alpha_{0} u_{1}(\mathbf{x}, t) u_{2}(\mathbf{x}, t) d \mathbf{x}-\frac{1}{2} \int_{\Omega} \int_{0}^{\infty}\left[k^{\prime}(\tau)\right]^{-1}\left[\mathbf{I}_{1}^{t}(\tau)\right]^{\prime} \cdot\left[\mathbf{I}_{2}^{t}(\tau)\right]^{\prime} d \tau d \mathbf{x},
$$

where $\mathbf{I}_{k}^{t}(\tau), k=1,2$, denote $\mathbf{I}\left(\tau, \overline{\mathbf{g}}_{k}^{t}\right)$ and the induced norm, which can be written as

$$
\|\sigma(t)\|^{2}:=\frac{1}{2} \int_{\Omega} \alpha_{0}[u(\mathbf{x}, t)]^{2} d \mathbf{x}-\frac{1}{2} \int_{\Omega} \int_{0}^{\infty}\left[k^{\prime}(\tau)\right]^{-1}\left\{\left[\mathbf{I}^{t}(\tau)\right]^{\prime}\right\}^{2} d \tau d \mathbf{x} .
$$

Accordingly, the energy functional associated to the rigid heat conductor can be defined in terms of the free energy $\Psi_{I}$ via

$$
\mathcal{E}(t)=\frac{1}{2} \int_{\Omega} \alpha_{0}[u(\mathbf{x}, t)]^{2} d \mathbf{x}-\frac{1}{2} \int_{\Omega} \int_{0}^{\infty}\left[k^{\prime}(\tau)\right]^{-1}\left\{\left[\mathbf{I}^{t}(\tau)\right]^{\prime}\right\}^{2} d \tau d \mathbf{x} .
$$

When (3.4) is satisfied, solutions to (4.21)-(4.22) exhibit an exponential decay at infinity. 
Lemma 4.3. Under the hypothesis (3.4), the total energy associated to the system (4.21)(4.22) is integrable on $[0, \infty)$.

Proof. Let $\sigma(t)$ denote a solution of the evolution problem (4.21)-(4.22); on substitution of such a solution into (1.15), recalling (4.16) and (4.17), integration over the whole space domain $\Omega$ shows that the time derivative of the free energy associated to the rigid heat conductor is nonincreasing; indeed, (4.17) implies that

$$
\int_{\Omega} \frac{d \Psi_{I}}{d t} d \mathbf{x} \leq 0
$$

where the r.h.s. is negative, as follows from (3.4). On the other hand, by definition (3.8), the initial value of the energy is given by

$$
\mathcal{E}(0)=\frac{1}{2} \int_{\Omega} \alpha_{0}\left[u_{0}(\mathbf{x})\right]^{2} d \mathbf{x}-\frac{1}{2} \int_{\Omega} \int_{0}^{\infty}\left[k^{\prime}(\tau)\right]^{-1}\left\{\left[\mathbf{I}^{0}(\tau)\right]^{\prime}\right\}^{2} d \tau d \mathbf{x} .
$$

Hence, it follows that the energy $\mathcal{E}(t)$, given by (4.25), satisfies

$$
0 \leq \mathcal{E}(t) \leq \mathcal{E}(0) \text {. }
$$

Moreover (4.26), on integration over $[0, \infty)$, gives

$$
\int_{0}^{\infty} \int_{\Omega} \frac{d \Psi_{I}}{d t} d \mathbf{x} d t \leq 0
$$

Furthermore, the same way of reasoning in Section 4 induces us to state Lemma 4.3 as well as Lemma 3.4, finally, Theorem 3.3 in the case of system (4.21)-(4.22) can be stated.

Notably, the function space for such a decay is established in the space $\mathcal{D}_{I}$, given by (4.18), is wider than the $\mathcal{D}_{G}$ in (3.15); hence, the result has a more general validity.

Acknowledgments. We gratefully acknowledge the partial support of the Italian G.N.F.M.-I.N.D.A.M. (National Research Group of Mathematical Physics - National Institute of "High Mathematics"), of SAPIEnZA, University of Rome, Research Project "Materiali speciali" e sistemi complessi: equazioni di evoluzione lineari e non lineari and of PRIN2005 Mathematical models and methods in continuum physics.

\section{REFERENCES}

[1] M.J. Ablowitz, A.S. Fokas, Complex Variables: Introduction and Applications, Cambridge University Press, Cambridge, 2003. MR 1989049 (2004f:30001)

[2] G. Amendola, S. Carillo, Thermal work and minimum free energy in a heat conductor with memory, Quart. J. of Mech. and Appl. Math. 57(3) (2004), pp. 429-446. MR2088844 (2005f:80002)

[3] C. Cattaneo, Sulla conduzione del calore, Atti Sem. Mat. Fis. Università Modena 3 (1949), pp. 83-101. MR0032898 (11:362d)

[4] S. Carillo, Some remarks on materials with memory: heat conduction and viscoelasticity, J. Nonlinear Math. Phys. 12 (2005), suppl. 1, pp. 163-178. MR2117178(2005h:74017)

[5] B.D. Coleman, Thermodynamics of materials with memory, Arch. Rat. Mech. Anal. 17 (1964), pp. 1-46. MR0171419 (30:1650)

[6] B.D. Coleman, E.H. Dill, On thermodynamics and stability of materials with memory, Arch. Rat. Mech. Anal. 51 (1973), pp. 1-53. MR0342046 (49:6792)

[7] R. Datko, Extending a theorem of A. M. Liapunov to Hilbert spaces, J. Math. Anal. Appl. 32 (1970), pp. 610-616. MR0268717 (42:3614) 
[8] M. Fabrizio, G. Gentili, D.W. Reynolds, On rigid heat conductors with memory, Int. J. Eng. Sci. 36 (1998), no. 7-8, pp. 765-782. MR.1629806 (99i:80006)

[9] M.E. Gurtin, A.C. Pipkin, A general theory of heat conduction with finite wave speeds, Arch. Rat. Mech. Anal. 31 (1968), no. 2, pp. 113-126. MR1553521

[10] M. McCarthy, Constitutive equations for thermomechanical materials with memory, Int. J. Eng. Sci. 8 (1970), pp. 467-474.

[11] S. Salsa, Partial Differential Equations in Action. From Modelling to Theory, Universitext. Springer-Verlag Italia, Milan, 2009. MR2399851 (2009b:35001)

[12] A. Pazy, Semigroups of linear operators and applications to partial differential equations, Applied Mathematical Sciences, 44, New York: Springer-Verlag, 1983. MR710486 (85g:47061) 